\title{
G6PD Deficiency and Priapism: Is There a Link Between the Two Entities?
}

\author{
Muhammad Muftia, d, Laura Alvarez ${ }^{\mathrm{a}}$, Omkar Marathe ${ }^{\mathrm{b}, \mathrm{c}}$
}

\begin{abstract}
Glucose 6 phosphate dehydrogenase (G6PD) is called the house keeping enzyme essential for all cells survival. G6PD deficiency is best studied for its role in hemolysis as this enzyme is the only source of nicotinamide adenine dinucleotide phosphate (NADPH) in red blood cells (RBCs) and is the cells defense against reactive oxygen species. We present one of the rare complications of G6PD deficiency in this case where all other causes of priapism were ruled out.
\end{abstract}

Keywords: Glucose 6 phosphate dehydrogenase deficiency; Sickle cell trait; Priapism; G6PD-induced priapism; NADPH

\section{Introduction}

The enzyme glucose 6 phosphate dehydrogenase (G6PD) is an essential enzyme for all cells of the body. For the red blood cells (RBCs), it is the first line of defense against reactive oxygen species. G6PD deficiency is known to cause hemolytic anemia but, in the case described below, we present a much rarer potential side effect of deficiency of this enzyme.

\section{Case Report}

A 48-year-old Caucasian man with a history of G6PD deficiency presented to the emergency department with 2 days history of sustained penile erection. He reported a sudden onset of erection non-related to sexual stimulation, which remained sustained, associated 10/10 pain. He tried gabapentin and acetaminophen for pain at home with no relief. He had one prior similar episode a year ago, and the erection lasted for 6

Manuscript submitted August 12, 2019, accepted August 19, 2019

aDepartment of Medicine, St. Mary Medical Center, Long Beach, CA, USA ${ }^{b}$ Department of Hematology/Oncology, St. Mary Medical Center, Long Beach, CA, USA

${ }^{\mathrm{c}}$ Oncology Institute of Hope and Innovation, Lakewood, CA, USA

${ }^{\mathrm{d} C}$ Corresponding Author: Muhammad Mufti, St. Mary Medical Center, GME, 1050 Linden Ave., Long Beach, CA 90813, USA.

Email: muneebmufti71@gmail.com

doi: https://doi.org/10.14740/jmc3355 $\mathrm{h}$ but resolved in the hospital without intervention. The patient denied urogenital trauma. He reported no use of any triggering medication such as a phosphodiesterase inhibitor, intracavernosal agent or any other medication. He reported a chronic history of hemolysis and anemia since childhood, which required multiple transfusion, and stabilized during adulthood. But he required splenectomy 2 years ago, and he was not sure about the reason for procedure. He also reported same hematological condition, G6PD deficiency in a male cousin.

On examination, patient was in pain, and his penis was erect, with no color changes suggestive of ischemia or necrosis, as well as no temperature change. Initial laboratory results showed macrocytic anemia (hemoglobin $(\mathrm{Hb}) 9.9 \mathrm{~g} / \mathrm{dL}$ and mean corpuscular volume (MCV) $123 \mathrm{fL}$ ), leukocytosis with white blood cell (WBC) 34,000/ $\mu \mathrm{L}$ (Diff Bands 10\%, Metas $1 \%$, Myelos $1 \%$, three nucleated RBCs), reticulocytosis, Ret $14.5 \%$; indirect hyperbilirubinemia, total bilirubin $2.6 \mathrm{mg} / \mathrm{dL}$, indirect bilirubin $1.0 \mathrm{mg} / \mathrm{dL}$; lactate dehydrogenase (LDH) $177 \mathrm{IU} / \mathrm{L}$, and haptoglobin $162 \mathrm{mg} / \mathrm{dL}$. Direct antiglobulin test (DAT) was negative. Peripheral blood smear showed presence of Howell jolly bodies and anisocytosis, and no blasts were seen. Toxicology test was negative except for opioids (urine morphine), and urine was collected after patient received morphine in the emergency department.

In the emergency department, patient had three rounds of drainage from either side of his cavernosum; during the second and third rounds, he was given $1 \mathrm{mg}$ of phenylephrine bilaterally. After procedure with persistent priapism, he was admitted for further urology management. During first day of admission, he was taken to operating room (OR) and underwent drainage of stagnant blood from the corpora cavernosa, injection of three syringes of epinephrine with saline into corpora cavernosa and manual reduction of priapism, with some pain relief and reduction of priapism, but after few hours of procedure, patient had persistent priapism. Hematology was consulted to evaluate for possible hematological conditions causing persistent priapism. No hemoglobinopathies were found, and hemoglobin electrophoresis showed no hemoglobin variants. No myeloproliferative process was identified, JAK 2 mutation was not detected, and BCR-ABL was negative. No other causes for hemolysis were recognized: paroxysmal nocturnal hemoglobinuria (PNH) and cold agglutinin were negative, and no reduction in RBC surface band 3 fluorescence for hereditary spherocytosis was detected. G6PD level was found to be low, and folate levels were depleted $(12.9 \mathrm{ng} / \mathrm{mL})$.

Patient was taken to OR on day 5 of admission for bilateral spongio-cavernosum shunts, and procedure was unevent- 
Table 1. Pertinent Labs

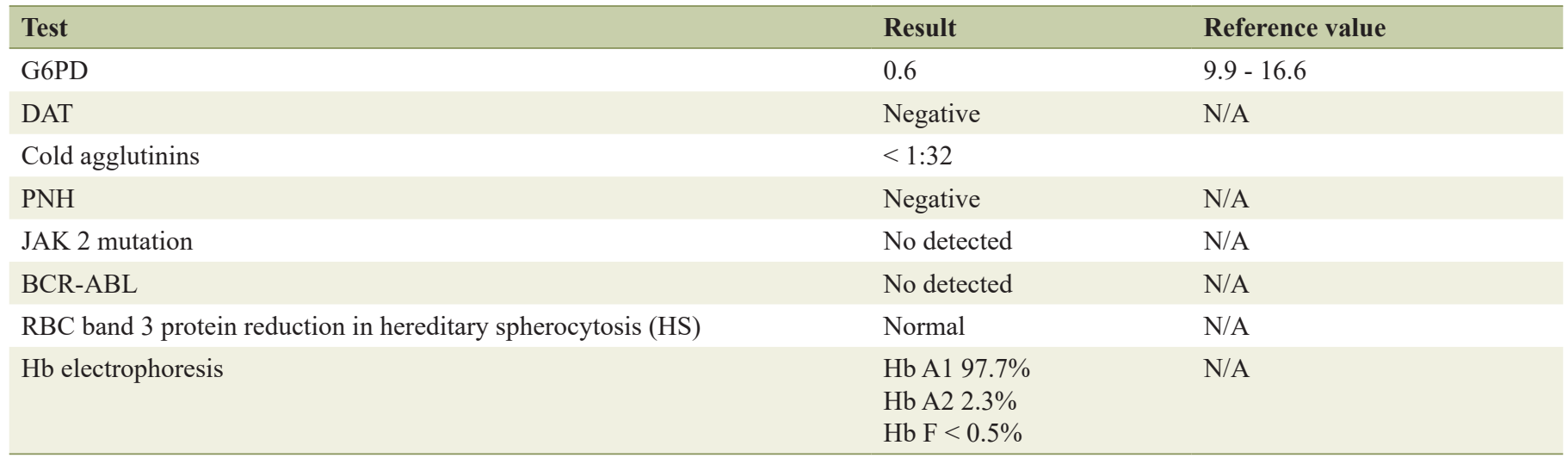

G6PD: glucose 6 phosphate dehydrogenase; DAT: direct antiglobulin test; PNH: paroxysmal nocturnal hemoglobinuria; RBC: red blood cell; Hb: hemoglobin.

ful. Patient had clinical improvement, with resolved priapism, and controlled pain. He was discharged from hospital on day 6 .

\section{Discussion}

G6PD is called the house keeping enzyme essential for all cells survival. The two key processes performed by this enzyme include the conversion of glucose 6 phosphate to 6-phosphogluconlactone and reduction of NADP to NADPH. G6PD deficiency is best studied for its role in hemolysis as this enzyme is the only source of NADPH in RBCs and is the cells defense against reactive oxygen species. Patients usually present with acute hemolytic anemia after oxidative stress.

This case highlights one of the rare complications associated with G6PD deficiency. This is to date the fourth known case on priapism with G6PD deficiency with all other possible causes for priapism ruled out. Priapism is usually associated with diseases like sickle cell or leukemia. The mechanism proposed with sickle cell is the nitric oxide, cyclic guanosine monophosphate (cGMP) and phosphodiesterase (PDE) signaling due to reduced tonic endothelial nitric oxide release [1]. Literature review suggests that NADPH oxidase is one of the mediators of priapism $[2,3]$. Morrison et al have proposed a possible mechanism of priapism that in G6PD deficiency oxidative stress which leads to hemolysis, endothelial injury and nitric oxide depletion. NADPH levels are reduced which causes reduced and aberrant nitric oxide signaling [4]. In most situations of oxidative stress when patients with G6PD deficiency present with acute hemolysis, the levels of G6PD enzyme are usually normal as the cells with low levels of the enzyme have lyzed but interestingly all of the cases described in literature who had priapism with G6PD desease had low G6PD levels but had no signs of acute hemolysis at the time of presentation [4-6].

Our patient when he presented had a sustained erection for 2 days. He had three rounds of drainage without success. His hematological workup included looking for hemoglobinopathies and none were found. Hemoglobin electrophoresis showed no hemoglobin variants. No myeloproliferative process or leukemia was identified, JAK 2 mutation was not detected, and BCR-ABL was negative. No other causes for hemolysis were recognized: $\mathrm{PNH}$ and cold agglutinin were negative, and no reduction in RBC cell surface band 3 fluorescence for hereditary spherocytosis was detected. Patient G6PD level was found to be low, and folate levels were depleted (full set of pertinent labs in Table 1). The limitations of our case include the fact that is not a well understood or proven consequence of G6PD deficiency and very few cases have been reported in literature. Secondly arterial blood gas analysis was not performed on the corporeal aspirate of our patient. More studies need to be done to further understand the processes involved in ischemic priapism in relation to G6PD deficiency.

\section{Acknowledgments}

None to declare.

\section{Financial Disclosure}

None to declare.

\section{Conflict of Interest}

None to declare.

\section{Informed Consent}

Patient's permission was taken so that his unique case could be shared with rest of scientific peers without identifying information.

\section{Author Contributions}

All of the authors performed literature review for the case re- 
port. Dr. Alvarez composed the case section and formulated the pertinent labs table. Dr. Mufti formulated the abstract and discussion section. Dr. Marathe reviewed the full case report and made corrections/suggestions which were implemented. He provided guidance on the disease etiology and pathogenesis. He was also the consulting hematologist on the case.

\section{References}

1. Champion HC, Bivalacqua TJ, Takimoto E, Kass DA, Burnett AL. Phosphodiesterase-5A dysregulation in penile erectile tissue is a mechanism of priapism. Proc Natl Acad Sci U S A. 2005;102(5):1661-1666.

2. Lagoda G, Sezen SF, Cabrini MR, Musicki B, Burnett AL. Molecular analysis of erection regulatory factors in sickle cell disease associated priapism in the human penis. J Urol. 2013;189(2):762-768.

3. Musicki B, Liu T, Sezen SF, Burnett AL. Targeting NADPH oxidase decreases oxidative stress in the transgenic sickle cell mouse penis. J Sex Med. 2012;9(8):19801987.

4. Morrison BF, Thompson EB, Shah SD, Wharfe GH. Ischaemic priapism and glucose-6-phosphate dehydrogenase deficiency: a mechanism of increased oxidative stress? West Indian Med J. 2014;63(6):658-660.

5. Finley DS. Glucose-6-phosphate dehydrogenase deficiency associated stuttering priapism: report of a case. J Sex Med. 2008;5(12):2963-2966.

6. Burnett AL, Bivalacqua TJ. Glucose-6-phosphate dehydrogenase deficiency: an etiology for idiopathic priapism? J Sex Med. 2008;5(1):237-240. 